\title{
Analysis of VR Sickness and Gait Parameters During Non-Isometric Virtual Walking with Large Translational Gain
}

\author{
Carlos A. Tirado Cortes \\ Carlos.TiradoCorts@uts.edu.au \\ Centre for Artificial Intelligence \\ Sydney, New South Wales
}

\author{
Hsiang-Ting Chen ${ }^{*}$ \\ Tim.Chen@uts.edu.au \\ Centrefor Artificial Intelligence \\ Sydney, New South Wales
}

\author{
Chin-Teng Lin \\ Chin-Teng.Lin@uts.edu.au \\ Centre for Artificial Intelligence \\ Sydney, New South Wales
}

\begin{abstract}
The combination of room-scale virtual reality and non-isometric virtual walking techniques is promising-the former provides a comfortable and natural VR experience, while the latter relaxes the constraint of the physical space surrounding the user. In the last few decades, many non-isometric virtual walking techniques have been proposed to enable unconstrained walking without disrupting the sense of presence in the VR environment. Nevertheless, many works reported the occurrence of VR sickness near the detection threshold or after prolonged use. There exists a knowledge gap on the level of VR sickness and gait performance for amplified nonisometric virtual walking at well beyond the detection threshold. This paper presents an experiment with 17 participants that investigated VR sickness and gait parameters during non-isometric virtual walking at large and detectable translational gain levels. The result showed that the translational gain level had a significant effect on the reported sickness score, gait parameters, and center of mass displacements. Surprisingly, participants who did not experience motion sickness symptoms at the end of the experiment adapted to the non-isometric virtual walking well and even showed improved performance at a large gain level of $10 x$.
\end{abstract}

\section{CCS CONCEPTS}

- Human-centered computing $\rightarrow$ Virtual reality; Mixed / augmented reality.

\section{KEYWORDS}

Virtual Reality, Cybersickness, Locomotion, Walking, Navigation, Redirected Walking

\section{ACM Reference Format:}

Carlos A. Tirado Cortes, Hsiang-Ting Chen, and Chin-Teng Lin. 2019. Analysis of VR Sickness and Gait Parameters During Non-Isometric Virtual Walking with Large Translational Gain. In The 17th International Conference on Virtual-Reality Continuum and its Applications in Industry (VRCAI '19), November 14-16, 2019, Brisbane, QLD, Australia. ACM, New York, NY, USA, 10 pages. https://doi.org/10.1145/3359997.3365694

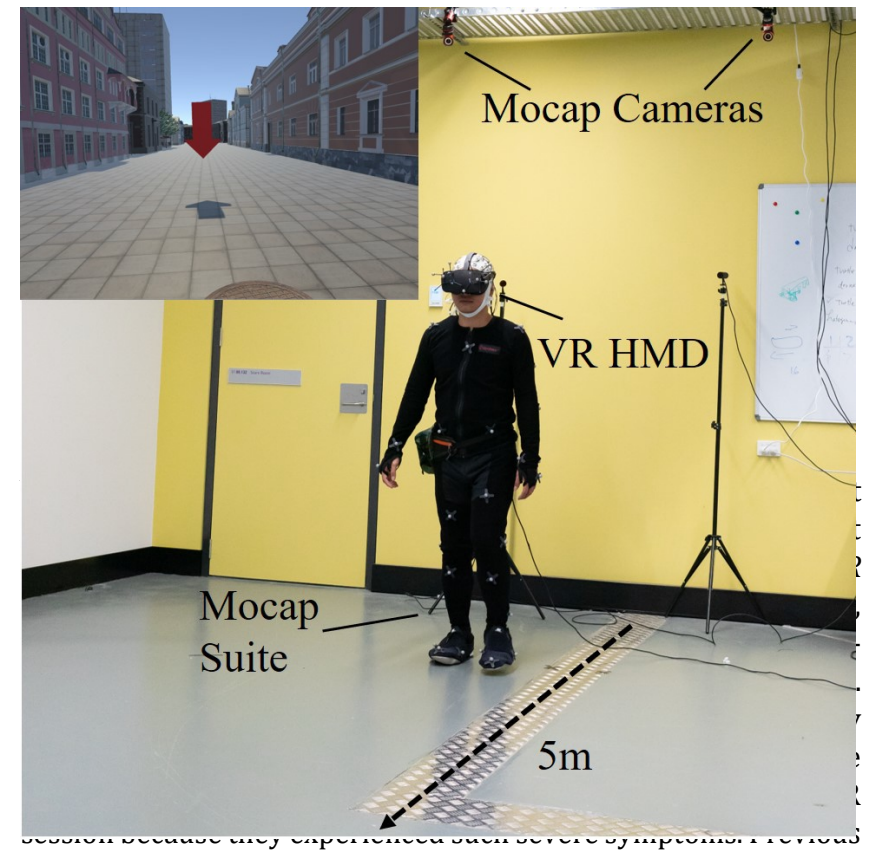

research [Nilsson et al. 2014, 2018; Usoh et al. 1999] reported that repeated exposure to the virtual environment could increase users' resilience to VR sickness. However, experiencing the discomfort of VR sickness might discourage even the most curious users from ever using VR again.

The combination of the room-scale VR and redirected walking techniques have received much attention in the research community. The high-precision and low-latency room-scale head tracking system enables the rendering of correct parallax effects as well as the navigation of the virtual environment through natural walking, 
which minimizes the potential sensory conflicts between a user's visual perception of the virtual environment and the sensory input of their vestibular system [Nilsson et al. 2018; Usoh et al. 1999]. The redirected walking techniques overcome the constraint of the physical space around the users and extend the coverage of the virtual space by adaptively amplifying or warping the mapping between the real and virtual space without users' awareness [Langbehn et al. 2017; Steinicke et al. 2010; Suma et al. 2012; Sun et al. 2016; Wilson et al. 2018]. Sun et al. [Sun et al. 2016] combined the detection of saccadic suppression and redirection techniques and achieved an impressive result of mapping a confined $3.5 \mathrm{~m} \times 3.5 \mathrm{~m}$ real room to a much larger $6.4 \mathrm{~m} \times 6.4 \mathrm{~m}$ synthetic virtual space.

Few studies have systematically investigated VR sickness induced by redirected walking techniques. We believe that the low quantity of work is due to dedicating research efforts more into to finding a balance between the intensity of perception manipulation and the probability of users' noticing the underlying alteration of the virtual space [Grechkin et al. 2016; Nilsson et al. 2018; Steinicke etal. 2010]. Multiple works [Janeh etal.2017a; Wilson etal.2018] have reported performance decreases and the occurrence of motion sickness symptoms at the gain value range between $1.5 X$ to $2.0 X$, where gain value represents the mapping between the physical and the virtual world. However, there is a knowledge gap regarding the relationship between the level of gain values and the severity of VR sickness, especially at a larger gain value. We argue that redirected walking techniques with larger, and noticeable, gain values are worthwhile. For example, a user might find virtual walking with a large translational gain more natural than other navigation metaphors such as teleportation, flying, or moving on a belt [Nilsson et al. 2018]. A user might also tolerate a temporary break of the sense of presence in exchange for traveling faster via natural walking in the virtual environment. We believe understanding the impact of non-isometric virtual walking techniques at a broader range of gain values - even well beyond the detection threshold [Grechkin etal.2016]-will enable a more comprehensive integration of redirected walking into different navigation techniques in VR.

This paper presents an experiment that investigated VR sickness, gait parameters, and posture instability during the non-isometric virtual walking experience, particularly at large and detectable translational gain (TG) levels. Figure 1 shows the experiment setup. The participant wore a VR head-mounted display (HMD) and a full-body motion capture suit, which enabled the recording of gait parameters (i.e., stepping distance and cadence) and center of mass (CoM) displacement. During the experiment, the participant was instructed to walk toward a destination marked by a red arrow and back to the original position on avirtualstreetin the city ofSydney with six different levels of translational gain at an increasing order $\left\{, 2,4,6,8,10^{\}}\right.$(Figure 2). We hypothesized that TG would have a main effect on the perceived level of VR sickness and CoM displacement and gait performance. More specifically, we hypothesized that participant would experience more severe VR sickness symptoms, larger posture instability, and decreased gait performance as TG increases.
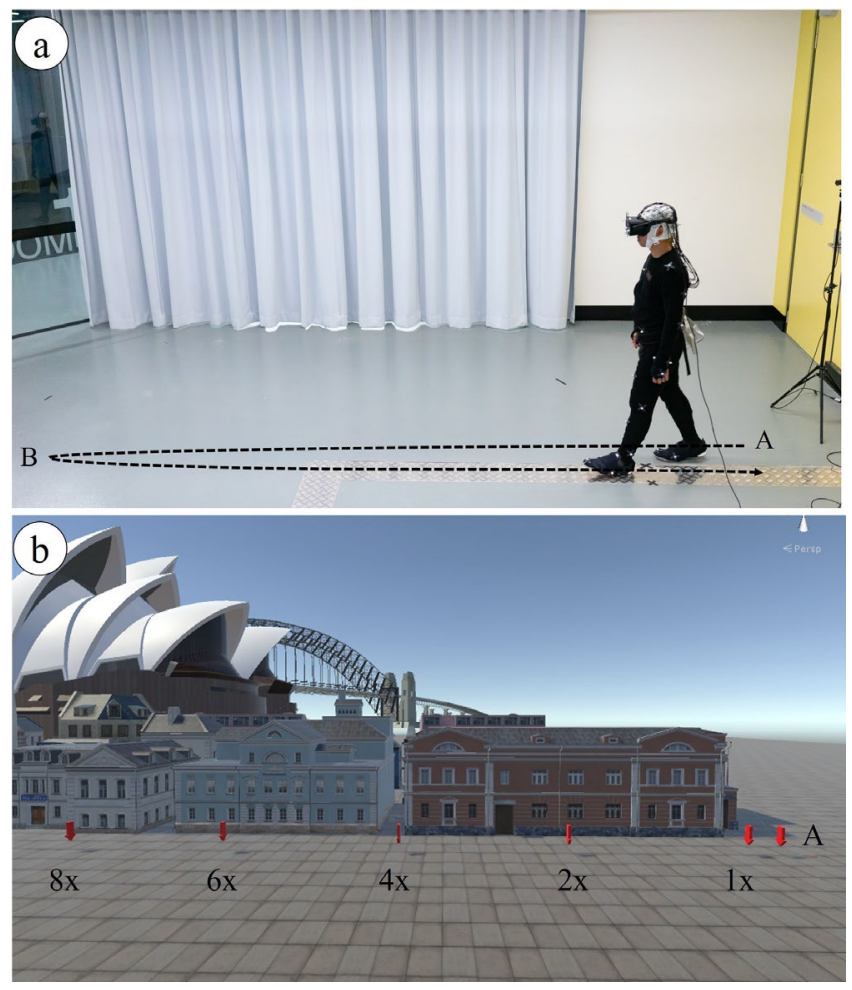

Figure 2: (a) Physical environment for the experiment, and (b) corresponding virtual environment with red arrows indicating the end point of the trials at different translational gains.

\section{RELATED WORKS}

\subsection{VR Sickness}

Despite the advancement of head-mounted display hardware, a large portion of VR users still experiences VR sickness symptoms such as headaches, nausea, vomiting, drowsiness, and disorientation [Davis et al. 2014]. Stanney et al. [Stanney et al. 1997] reported that compared to traditional motion sickness, VR sickness can be three times more severe and exhibits a different symptom profile. Among various theories for the cause of VR sickness [LaViola 2000], the sensory conflict theory has been the most widely accepted [Kennedy etal.1993; LaViola 2000]. The sensory conflict theory argues that the cause of VR sickness is the conflict between sensory input systems engaged in the virtual environment. For example, when experiencing a VR roller coaster in a stationary setup, a sensory conflict arises because the visual system perceives a forward-moving optical flow pattern, while the vestibular system does not sense a proportional linear or angular motion. Another competing theory for VR sickness focused on the postural instability and argued that decreased postural stability magnifies cue-conflicts that underlie sickness symptoms [Chardonnet et al. 2017; Riccio and Stoffregen 1991].

The research community has long been aware of VR sickness [Davis et al. 2014; Duzmanska et al. 2018; Rebenitsch and Owen 2016], and has proposed a stream of creative methods to reduce the 
sickness symptoms [Argelaguet 2014; Fernandes and Feiner 2016; Palmisano et al. 2011; Tregillus and Folmer 2016]. Many preventative approaches reduce the occurrence of sensory mismatch. These approaches include navigating a virtual environment with a point and teleportation method that relocates the user immediately to the selected destination [Bozgeyikli et al. 2016]. Applying blurring [Patney et al. 2016] and vignetting [Fernandes and Feiner 2016] in peripheral vision, which has high motion sensitivity, to reduce the perceived visual motion. Another prominent technique is galvanic vestibular stimulation (GVS) which applies an electrical current to stimulate vestibular afferent nerves and recouple visual and vestibular cues [Cevette et al. 2012; Gálvez-García et al. 2015; Zao et al. 2016]. Notably, most of these previous works focused on stationary VR setups. The users usually remained relatively stationary on a seat where visual motion patterns on 2D displays or head-mounted displays created sensory conflicts.

\subsection{Redirected Walking}

Natural walking in VR provides a superior VR experience, yet the physical space around the user constrains the traversable area. Nilsson et al. [Nilsson et al. 2018] categorized techniques for overcoming the physical space constraint for natural walking into three classes: repositioning, proxy gestures, and redirection techniques. Repositioning techniques leverage different types of treadmill systems, such as motorized treadmills [Iwata et al. 2005] and frictionless omnidirectional treadmills [Swapp et al. 2010]. These treadmills are used to offset the user's forward movement and keep the user at the same position. Proxy gestures techniques drive movements in the virtual environment through proxy gestures resembling real walking, such as upper arm-waving [McCullough et al. 2015; Nilsson et al. 2013], head tilt [Tregillus et al. 2017], and walk-in-place [Slater et al. 1995]. Redirection techniques manipulate the user's actual walking path in the real world, without being perceived by the user, to exploit the limited physical space. Path manipulation is achieved either by dynamically scaling user motion [Steinicke et al. 2010; Wilson et al. 2018], or by updating the virtual environment [Baur et al. 2012; Garrett et al. 1988; Suma et al. 2012; Sun et al. 2016].

This paper investigates the redirection technique that extends the physical space by amplifying the mapping between physical and virtual movement, which is called translational gain. This technique is popular in VR [Agethen et al. 2018; Interrante et al. 2007; Janeh etal. 2017b; Ragan et al. 2017; Steinicke etal. 2010] because of its simplicity and its preservation of the natural walking and vestibular self-motion information. Previous papers have examined the impact of the translational gain on gait parameters [Janeh et al. 2017b] as well as on object selection performance [Wilson et al. 2018]. Proof exists that there is a detection threshold around $1.25 \mathrm{x}$ for translational amplification and $1.5 \mathrm{x}$ for rotational amplification [Grechkin et al. 2016; Steinicke et al. 2010]. However, it is unclear how exposure to substantial perceivable translational gains would affect the user's perception and gait performance.

\section{EXPERIMENT}

Our experiment investigated the correlation between the level of translational gain of virtual walking and the severity of VR sickness.
Complementing previous works that examined the usability of translational gain ranges below 3x [Agethen et al. 2018; Interrante et al. 2007; Janeh et al. 2017b; Ragan et al. 2017; Steinicke etal. 2010; Wilson et al. 2018], our experiment examined a more extensive gain range from 1 to 10 and focused on how VR sickness affected the behavior of the users performing virtual walking while wearing an HMD.

\subsection{Participants}

Twenty-one healthy adults (17 males, four females) participated in the experiment. The mean age was 25.73 , with a standard deviation of 3.594. All participants were paid for their participation and gave written informed consent. All participants had a normal or corrected-to-normal vision. We encouraged participants to wear contact lenses for a more comfortable Oculus VR experience. Among all participants, 13 had prior experience with threedimensional computergames, and 9 had previous experience on VR; 15 had experienced motion sickness of different severity previously in their life.

\subsection{Physical Space and Virtual Environment}

The physical space of the experimental environment was three by 5 meters. In each trial, the user was instructed to walk from point A to point $B$ and back to point $A$ (Figure $2 \mathrm{a}$ ). We marked both points $A$ and $B$ with black tape on the ground. The distance between $A$ and $B$ was $4.5 \mathrm{~m}$, which can be covered within eight steps by a 175 $\mathrm{cm}$ male adult.

The corresponding virtual space to the lab environment was a virtual streetscene. The size of the whole Sydney scene was $100 \mathrm{~m}$ by $100 \mathrm{~m}$. The virtual walking took place on a straight street of 70 $\mathrm{m}$ long and $5 \mathrm{~m}$ wide. Figure $2 \mathrm{~b}$ shows the side view of the street, and Figure 1 shows the first-person view of the participant while performing the walking task.

To achieve the different levels of translational gain, we modified the translation matrix of the entire Sydney scene in the opposite direction of the movements of the user. Note that we modified the translation of the scene towards all the directions, not only the forward direction. We decided that such a change will help us create a noticeable conflict that will end up introducing VR sickness easier.

\subsection{Experiment Design}

The experiment used a within-subject design with translational gain as the sole independent variable, with six levels $\{1,2,4,6,8,19$. An informal internal pilot test led to our decision to set $10 x$ gain as the upper bound of the experiment. VR engineers on our team, who were used to different redirected walking experiments, all considered the virtual walking experience uncomfortable and unusable at gain levels of 10 and above.

The participants experienced five trials per gain level, for a total of 30 trials in increasing order from $1 x$ to $10 x$ during the experiment session. We chose not to randomize the order of translational gain because we hypothesized that exposure to large translational gain combined with the gain applied to all axis, would induce severe VR sickness in some participants. We wanted to avoid these sickness symptoms persisting throughout the entire session. Using a fixed 
increasing order of translational gain also enabled us to investigate the habituation on the translational gain when the VR users were expecting an amplification of the mapping between the virtual and physical worlds.

At the beginning of each trial, the participant was instructed to move to a virtual utility hole in the virtual street (point A in Figure 2). She was then instructed to walk toward a destination indicated by a red arrow (Figure 1 ) in the virtual scene and then back to the virtual utility hole. As the translational gain increased, the position of the red arrow moved farther away from the starting point (Figure $2 \mathrm{~b})$. In the physical world, the participant walked between points $A$ and $B$ (Figure 2a). We implemented physical collision in the virtual environment to prevent the unlikely event of the user running into the virtual building.

\subsection{Apparatus}

- Motion Capture System. We used an OptiTrack motion capture system with 12 Flex 13 cameras for full-body tracking. Flex 13 captured information at a rate of 120 frames per second. Participants wore the motion capture suit over their clothing. The OptiTrack Unity Plugin synchronized the motion capture data and the coordinate systems in both the OptiTrack cameras and the Unity3D engine. In this experiment, we used the Baseline + Toe marker skeleton setup with 41 tracked markers in the Motive: Body software from OptiTrack. This setup enabled us to calculate biomechanical measurements, including CoM [Clauser, Charles E; McConville, John T; Young 1969; Lafond etal. 2004], stepping distance, and cadence.

- VR Headset. We used the Oculus VR CV1 headset in this experiment. We chose Oculus because of its compatibility with the OptiTrack Flex 13 cameras. The tracking information from the OptiTrack system overrode the head position of the Oculus VR plug-in in Unity3D, which enabled a larger walking area. The headset contained a pair of OLED displays that provided 110-degree field-of-view with a resolution of 1080 x 1200 pixels per eye.

\subsection{Procedure}

Before the experiment started, each participant answered a preexperiment questionnaire. This questionnaire was designed to provide an understanding of two critical elements for our experiment: their level of familiarity with 3D and VR technologies and their experience (if any) with motion sickness. Afterward, the participant first wore the motion capture suit and was instructed to freely walk in our tracking area to confirm that the motion capture cameras could track the full-body motion inside the tracking volume. Once the motion capture system was ready, the participant started five baseline walking trials without wearing the VR headset. The participant walked between the two ends of the walking area (i.e., positions A and B in Figure 2a). Afterward, the participant put on the VR headset and was introduced to the virtual environment and the task of starting at the starting point, walking toward the endpoint (i.e., a red arrow), and finally walking back to the starting point. This walk between starting and endpoints constituted a single trial. At the end of each trial, a virtual message would indicate the end of the trial, and the participant reported her level of sickness from 1 to 10 , where ten meant the sickness was so severe that the experiment should stop immediately.

\section{MEASUREMENTS}

- Between-TrialQuestionnaire. At the end of each trial, we asked the participants to express on a scale from 1 to 10 their feelings of dizziness, discomfort, nausea, fatigue, headache, and eyestrain. We chose these symptoms following previous works [Lin et al. 2013; Seay etal. 2002; Wilson et al. 2018], which also used a sub-set of symptoms in trials to avoid disrupting the immersion.

- Post-Experiment Questionnaire. Upon the completion of the VR session, the participant was asked to complete a full SSQ, followed by a semi-structured post hoc interview session where the researcher encouraged participants to think out loud about their experience and responses to the questionnaire.

- Center of Mass. To analyze the change in CoM, we calculated the displacement between the CoM measured during the baseline walking trials without the VR headset and the CoM measured during virtual walking at different TG. We followed the methodologies described by Lafond et al. [Lafond et al. 2004] to calculate CoM. The length of all trials was aligned by MATLAB's Dynamic Time Warping function to facilitate the calculation of average CoM displacement.

- Gait Parameters. Step distance was calculated based on the motion of the markers on the participant's feet. We followed the work by Hreljac et al. [Hreljac and Marshall 2000] to obtain the stepping information. We segmented each step by detecting changes in acceleration direction during walking. More specifically, we used the function called findpeaks.m from MATLAB. The MinPeakProminence parameter was set to 0.01 to remove all the noise peaks, leaving only those peaks that represented a significant movement of the feet.

\section{RESULTS}

On average, the entire experiment took about 25 minutes per participant. Among the 21 participants, four were removed: 2 due to tracking malfunction and 2 to software malfunction during the experiment. Out of the 17 remaining participants, 3 participants terminated the experiment before TG $10 x$ due to severe VR sickness symptoms at TG $4 x, 6 x$, and $8 x$ respectively.

\subsection{Questionnaire Responses}

Table 1 shows the results of the post-experiment questionnaire. Kennedy et al. [Kennedy et al. 1993; Stanney et al. 1997] suggested a threshold around 18 as an indicator of a problematic level of sickness. Eight out of 17 participants had a total severity (TS) score around or higher than 18, including three who quit the experiment prematurely (marked with an * in Table 1). To further investigate the relationship between VR sickness and gait parameter changes, we categorized those 8 participants with high TS scores into the motion sickness group (MS) and the remaining 13 participants into the no-motion sickness group (No-MS). In the following paragraphs, 
Table 1: Post-experiment SSQ results. Rows with * sign are participants who quit the experiment prematurely. SSQ-N is the nausea score, SSQ-O is the oculomotor score, SSQ-D is the disorientation score, and TS is the total score. Rows with red background color are participants in the MS group and rows with green background color are participants in the No-MS group.

\begin{tabular}{|c|c|c|c|c|}
\hline & SSQ-N & SSQ-O & SSQ-D & TS \\
\hline S1 & 19.08 & 7.58 & 55.68 & 17.96 \\
\hline S2* & 57.24 & 75.8 & 83.52 & 38.44 \\
\hline S3 & 9.54 & 0 & 13.92 & 4.74 \\
\hline S4 & 57.24 & 45.48 & 111.36 & 41.92 \\
\hline S5 & 0 & 0 & 0 & 0 \\
\hline S6 & 19.08 & 30.32 & 0 & 6 \\
\hline S7 & 28.62 & 22.74 & 27.84 & 13.48 \\
\hline S8 & 9.54 & 0 & 13.92 & 4.74 \\
\hline S9 & 38.16 & 22.74 & 125.28 & 40.66 \\
\hline S10 & 19.08 & 22.74 & 0 & 5 \\
\hline S11 & 0 & 0 & 0 & 0 \\
\hline S12 & 66.78 & 75.8 & 180.96 & 65.62 \\
\hline S13 & 28.62 & 7.58 & 55.68 & 18.96 \\
\hline S14* & 38.16 & 7.58 & 97.44 & 31.18 \\
\hline S15 & 0 & 0 & 0 & 0 \\
\hline S16* & 76.32 & 90.96 & 153.12 & 61.14 \\
\hline S17 & 28.62 & 0 & 41.76 & 14.22 \\
\hline
\end{tabular}

we analyze and report measurements for all participants, the MS group, and the No-MS group.

\subsection{Trial Questionnaire Results}

Out of the symptoms recorded, every participant recorded some levels of dizziness, discomfort, and nausea. Only 2 participants reported changes in eyestrain. Four participants reported an increase in fatigue (which they later confirmed was due to wearing the equipment and not because of the interaction with the scenario), and only 3 participants reported headache ( 2 of which, again, reported it was due to finding the VR headset uncomfortable and not due to interacting with the scenario). To visualize the between-trial questionnaire results, we averaged the responses from all symptoms at each trial. Those responses were then grouped per TG level for each participant and then averaged with the rest of the participants. Figure 3 shows the resulting values for the overall group, as well as for the MS and No-MS groups.

A Friedman test was used to test for the main effect of TG on the user questionnaire responses. We used a Wilcoxon Signed-rank

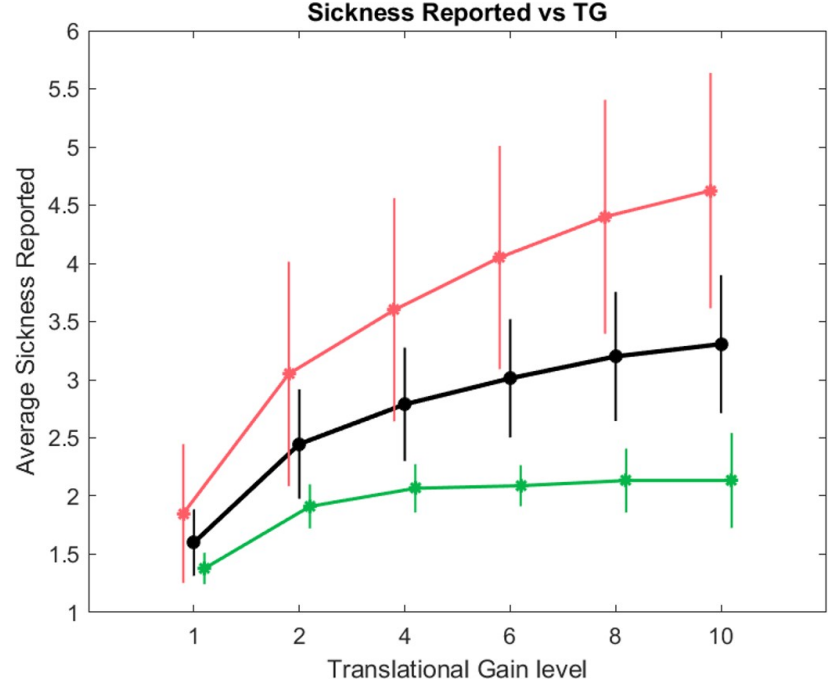

Figure 3: Average per-participant response to each TG level. Black Line: Reported sickness levels for all the participants. Red Line: Reported sickness levels for MS participants. Green Line: Reported sickness levels for No-MS participants.

test as a post hoc test in the case that there was any effect. For the overall data, the results showed that there was a significant effect of TG on the reported between-trial sickness scores. Results and post hoc tests are shown in Table 2, last column.

\subsection{Behavior and Gait Analysis}

The four measurements we analyzed were CoM displacement, stepping distance, cadence, and trial completion time. Figure 4 shows the changes in each of the measurements at the different levels of TG for all data, MS group, and No-MS group, respectively.

The first set of tests compared baseline normal walking without HMD against an isometric virtual walking with $1 x$ TG. Normality was tested in the three pairings (cadence, step distance, and trial time completion) using a Shapiro-Wilk test for normality. Cadence and trial completion time failed to follow a normal distribution while step distance followed a normal distribution. For data sets that did not follow a normal distribution, a Friedman test was used to see if there was an effect from walking with the VR headset. For data sets that followed a normal distribution, we used a repeated measure ANOVA with Greenhouse-Geisser correction. For cadence, we found that an effect existed $\left(x^{2}(1)=10.28, p=0.001\right)$. The test on stepping distance revealed that an effect existed $(k 1,2)=$ $61.54, p=0.000005)$. For trial completion time, there was an effect $\left(x^{2}(1)=4.571, p=0.033\right)$. Because our data for the center of mass already measures a difference between the TG levels and no-VR walking, we did not do this analysis for the center of mass.

The next set of tests compared measurements between different levels of TG. In the case that the data sets followed a normal distribution. Weused a repeated-measures ANOVA with a GreenhouseGeisser correction to test for effects. We chose a paired sample t-test as a post hoc. For data that does not follow a normal distribution, 
Table 2: Statistical results for the different measurements. The first 4 columns represent different behavior measurements. The last column represents the sickness level reported by each participant.

\begin{tabular}{|c|c|c|c|c|c|}
\hline & Cadence & CoM Displacement & Step Distance & Trial Completion Time & Reported Sickness Level \\
\hline \multirow[t]{2}{*}{ Overall } & $\begin{array}{c}x^{2}(5)=33.592 \\
p=0.000003\end{array}$ & $\begin{array}{l}x^{2}(5)=22.98 \\
p=0.000341\end{array}$ & $\begin{array}{c}F 2.775,36.074=6.077 \\
p=0.002\end{array}$ & $\begin{array}{c}x^{2}(5)=22.571 \\
p=0.000408\end{array}$ & $\begin{array}{c}x^{2} 5=41.274 \\
p<0.05\end{array}$ \\
\hline & $\begin{array}{c}\partial\{2,4,6,8,10\}-1 \\
\partial\{4,6\}-10\end{array}$ & $\begin{array}{c}\partial\{4,6,8,10\}-1 \\
\partial\{2,10\}-8\end{array}$ & $\begin{array}{c}\partial\{4,6,8,10\}-1 \\
\partial\{6,8\}-2\end{array}$ & $\partial\{2,4,6,8,10\}-1$ & $\begin{array}{c}\partial\{2,4,6,8,10\}-\{1,2,4\} \\
\partial\{8\}-6\end{array}$ \\
\hline \multirow[t]{2}{*}{ MS Group } & $\begin{array}{c}x^{2}(5)=15.971 \\
p=0.007\end{array}$ & $\begin{array}{c}F(.826,7.306=6.742 \\
p=0.023\end{array}$ & $\begin{array}{c}x^{2}(5)=18.029 \\
p=0.003\end{array}$ & $\begin{array}{c}F(.264,5.058=2.964 \\
p=0.145\end{array}$ & $\begin{aligned} x^{2}(5) & =37.491 \\
p & <0.05\end{aligned}$ \\
\hline & $\begin{array}{c}\partial\{2,6,8,10\}-1 \\
\partial\{6,8\}-4 \\
\partial\{8\}-2\end{array}$ & $\begin{array}{c}\partial\{4,6,8,10\}-1 \\
\partial\{10\}-8\end{array}$ & $\begin{array}{l}\partial\{6,8,10\}-1 \\
\partial\{4,6,8,10\}-2\end{array}$ & - & $\begin{array}{c}\partial\{1,2,4,6,8,10\}- \\
\partial\{2,4,6,8,10\}\end{array}$ \\
\hline \multirow[t]{2}{*}{ No-MS Group } & $\begin{array}{c}x^{2}(5)=21.317 \\
p<0.05\end{array}$ & $\begin{array}{c}F 2(461,19.687)=5.718 \\
p=0.008\end{array}$ & $\begin{array}{c}F 2.44,19.55=2.524 \\
p=0.097\end{array}$ & $\begin{array}{c}x^{2}(5)=14.651 \\
p=0.012\end{array}$ & $\begin{array}{c}x^{2}(5)=12.516 \\
p=0.028\end{array}$ \\
\hline & $\begin{array}{l}\partial\{2,4,6\}-1 \\
\partial\{4,6\}-10\end{array}$ & $\begin{array}{c}\partial\{4,6,8,10\}-1 \\
\partial\{4,6,8\}-2\end{array}$ & - & $\begin{array}{l}\partial\{2,4,6\}-1 \\
\partial\{10\}-6\end{array}$ & $\partial\{2,4,6,8\}-1$ \\
\hline
\end{tabular}

we used the Friedman test as a repeated measure test, and we used the Wilcoxon Signed-rank test as a post hoc. Table 2 summarizes the results.

5.3.I Cadence. We treated the data from the three groups as not following a normal distribution, and the repeated measure tests were used to see if there was any effect of TG levels on the cadence. The results showed that for all the groups there was an effect of TG on cadence. Column 1 in Table 2 shows the results of the post hoc tests.

5.3.2 Center of Mass Displacement. After doing a normality test on the three different groups of the center of mass difference, we treated the overall group as not following a normal distribution, while the MS and No-MS groups followed a normal distribution. For the overall group, the repeated measure test showed that there was an effect of TG levels on the difference in center of mass. Column 2 in Table 2 shows the results of the post hoc tests.

5.3.3 StepDistance. After running a normality test on the different step distance groups, the results showed that the overall group and the No-MS group followed a normal distribution and that the MS group did not follow a normal distribution. For the overall group, the repeated measures test showed that there was an effect from TG on step distance. Column 3 in Table 2 shows the results of the post hoc tests.

5.3.4 Trial Completion Time. For the trial completion time, the overall group and the No- MS group did not follow a normal distribution. The MS group followed a normal distribution. For the overall and No-MS group, the level of TG affected on trial completion time. Column 4 in Table 2 shows the results of the post hoc tests.

\section{INTERVIEW RESPONSES}

All participants except one (S2) reported that the symptoms significantly decreased after removing the headset at the end of the experiment. Out of the 17 participants, 8 participants reported that their level of sickness was partially due to the prolonged use of VR; 3 participants (S3, S9, S10) thought their sickness symptoms were mainly due to the sudden changes of TG across trials. Three participants reported having no symptoms after the experiment finished.

From the pre-experiment interviews, three participants (S2,S14, S16) reported high susceptibility to motion sickness. S2 and S16 stated that as soon as they wore the VR headset, they started to feel very uncomfortable. S2 had to stop the experiment at trial 27 (at the gain level of $8 \mathrm{x}$ ). S2 was also the only participant whose sickness symptoms did not diminish after removing the headset. S5, S11, and S15 did not report any symptoms after the experiment. S5 stated that she is so used to playing first-person shooting video games online that her experience with constant frame drops in the games prevented her from suffering any symptom. S11 mentioned that she suffers from motion sickness on the bus regularly. However, to her surprise, her VR sickness symptoms subsided once she removed the VR headset. S6 also expressed how each change in translational gain caused her surprise and how this surprise caused her to experience vertigo. S16 expressed her difficulty traveling on an airplane, because looking at the movement outside the window while sitting down causes her motion sickness. During the experiment, the participant expressed that after reaching level $2 x$, she started feeling the same symptoms. This participant dropped out of the experiment the earliest, quitting once the gain level changed from $2 \mathrm{x}$ to $4 \mathrm{x}$.

Out of the 17 participants, 5 participants (S6, S7, S10,S11,S13) reported thatafter reaching 15 to 16 trials, they could confidently prepare themselves for the next translational gain change, which 

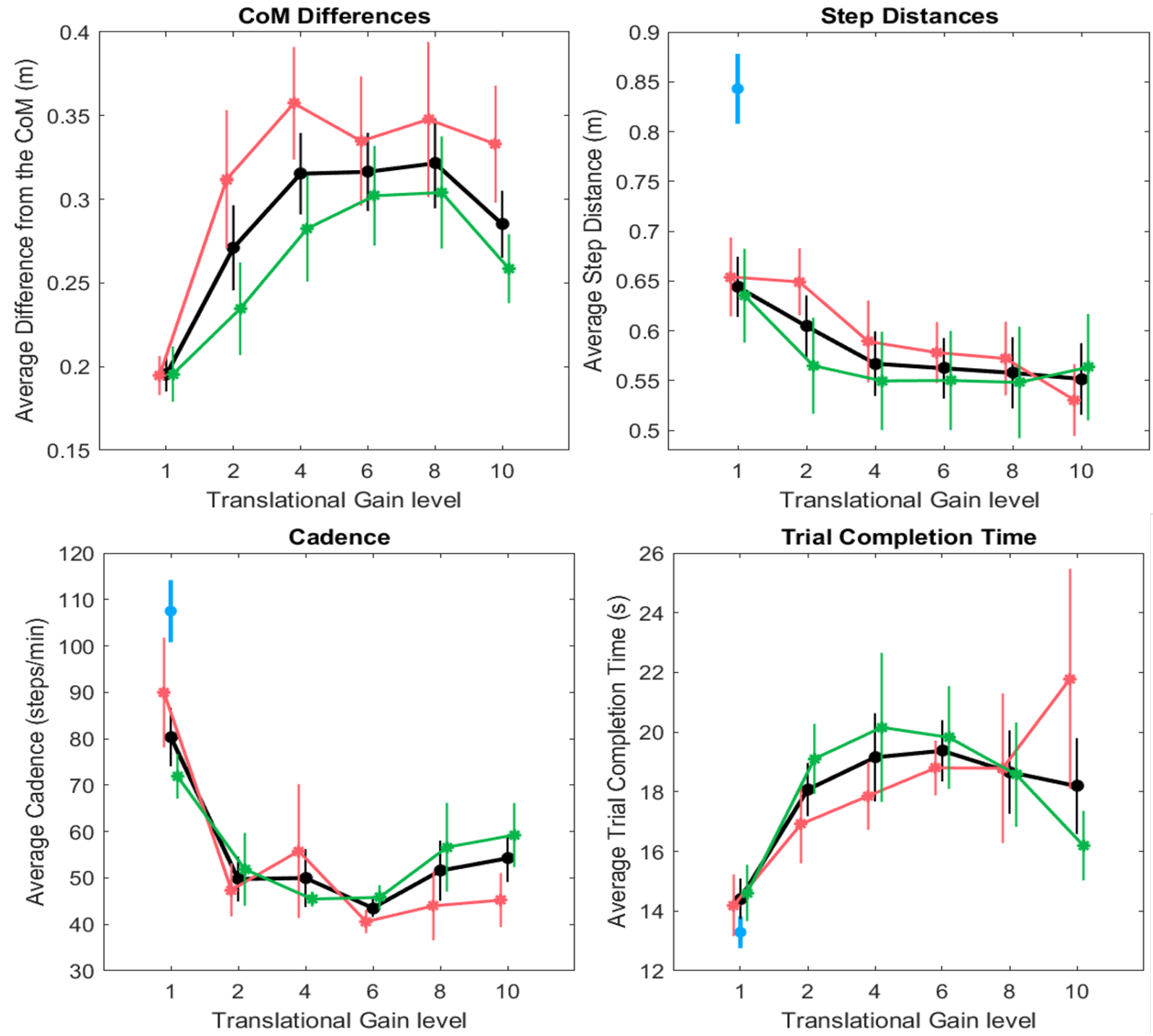

Figure 4: Results of different behavior measurements vs. different levels of TG. Black Line: Average. Red Line: Results from participants belonging to the MS group. Green Line: Results from participants in the No-MS group. Blue Line: Average baseline recording without VR. Position dodge function was used to avoid the overlapping of standard error bars.

helped in decreasing the level of dizziness. Contrary to these statements, 2 participants (S12, S14) expressed that five trials were not enough to get used to the translational gain and that the constant change in translational gain caused their symptoms. It is also worth noting that 2 out of the 17 participants (S13, S14) started to feel dizziness and nausea 20 minutes after the experiment ended, although they reported no symptoms at post-experiment SSQ.

\section{DISCUSSION}

The result showed that $\mathrm{TG}$ had main effects on the reported sickness level. In general, as the TG level increased, the reported sickness levels between the trials also increased (Figure 3). However, for the No-MS group, whose participants reported low severityscores in the post-experiment SSQ questionnaires, the between-trials VR sickness scores stayed low even after $2 x$ TG and showed no significant difference among the larger TG $\{4 x, 6 x, 8 x, 10\}$. This result seems to suggest that non-isometric virtual walking with a large TG could be an effective and practical navigation method for at least a sub-group of users in the scenario where precise destination selection is not required.

Note, one participant reported lower VR sickness at the end of the experiment, possibly because she was better able to adapt to the non-isometric virtual walking or because she was just more resilient to VR sickness in general. Given the fact that none of the participants had everexperienced non-isometric virtual walking 
before, in this case we would lean toward the latter assumption as being more likely.

We found that gait performance was significantly different between VR walking and the baseline non-VR walking, which concurred with the findings of Janeh et al. [Janeh et al. 2017a]. Among the VR walking trials, the most significant differences in gait performance and CoM displacement were between the $1 x$ and other TG levels. The difference was particularly prominent when the study participants first experienced an amplified TG at $2 x$, as shown in Figure 4. At $2 x \mathrm{TG}$, a sudden increase in CoM displacement happened, indicatinga decrease in posture stability; also, the corresponding gait patterns, namely a significantly smaller stepping distance and slower cadence, were signs of participants spending more than usual attentional resources trying to control their gait [Woollacott and Shumway-Cook 2002; Yogev-Seligmann et al. 2008]. These changes also led to a significantly longer trial completion time, even though the physical walking distance for each trial is the same.

At larger TG levels, namely $\{x, 6 x, 8 x, 10\}$, there were few pair-wise significant differences between the measurements. Surprisingly, the participants even on average performed slightly better at large TG $8 x, 10 x$ \}in some measurements, such as demonstrating smaller CoM displacements and a shorter task completion time. The participants in the No-MS group, i.e., those who did not report severe MS symptoms after the experiment, seemed to be able to adapt to the increase of TG particularly well and even started increasing their step distances and thus reducing the task comple-

tion time after $4 x$ TG. In contrast, participants in the MS group, i.e., those who had reported severe MS symptoms, struggled to perform the virtual walking task on higher levels of TG. With the latter group, all their performance measurements steadily decreased, even though at this stage, the participants had more experience with non-isometric virtual walking. The discomfort from their MS and other MS symptoms seemed to impede their ability to learn and adapt to virtual walking at larger TG levels.

In summary, non-isometric virtual walking with a large and detectable TG is a simple and straight-forward navigation technique that can be used to expand the coverage of a virtual environment within a restricted physical space. All the present study participants understood the concept quickly and could use the technique without any prior training. However, this navigation technique might not be suitable for all users. Some users, such as those in the No-MS group, could adapt to the technique quickly and achieve good performance even at high TG levels. Whereas some other users, such as those in the MS group, even with a clear expectation of the upcoming TG levels, the associated severe VR sickness symptoms could render the virtual walking at large TG levels undesirable. Understanding how to predict users' resilience to the effects of virtual walking with different TGs will be an important step toward promoting nonisometric virtual walking with large gains as a practical navigation method in the virtual environment.

\section{LIMITATION AND FUTURE WORK}

The presented experiment design used a single independent variable of translational gain. It would be interesting to investigate whether the results could be generalized for other types of redirected walking techniques, such as rotational and curvature gain. Previous works [Grechkin et al. 2016; Steinicke et al. 2010] found that users are more sensitive to translational gain than rotational gain, and so it would be interesting to test this statement at larger gain values and for people with different susceptibility to VR sickness.

Our experiment design did not address the potential confound of VR exposure time, i.e., the VR sickness and gait parameters changes were also affected by the continuous exposure to the virtual environment. We did a preliminarypost-hoc baseline experiment that recalled 4 participants ( 2 from both MS and No-MS group) to experienced all 30 trials using TG level 1 only. The result did not show significant changes in all gait parameters. We suspect this confound was also minimized due to our relatively short experiment sessions, i.e., 15 minutes on average, which was shorter than other redirected walking experiments [Janeh et al. 2017a; Steinicke et al. 2010] that range from 30 minutes to one and half hours. Nevertheless, we believe examining the interaction between VR exposure time and VR sickness during non-isometric walking is an interesting research direction.

Another limitation that this experiment presents is the application of TG on all the axis of the scenario and not just the forward axis. Unlike the works of [Janeh etal.2017a,b], where TG is applied only on the forward axis, we hypothesized that applying it to all the axis, we would be able to induce VR sickness quickly. It would be interesting to compare the results from the SSQ reported from participants walking on a scene with only forward TG applied, vs. walking on a scenario where that has TG applied on all axis.

Previous works reported that visualization of a virtual avatar would affect the level of a user's presence and induce different responses toward visual stimuli in the virtual environment [Banakou et al. 2013; Singh et al. 2018; Slater et al. 2009]. However, we opted not to show the virtual avatars because of the challenges involved in visualizing the locomotion animation correctly with the increase in the translational gain. At larger TG, an accelerated animation would create an illusion of sliding on the floor. Toavoid the risk of this confounding our participants, we decided to hide the avatar. Nevertheless, we believe it is an interesting research question, and future studies should investigate how to visualize the walking animation correctly when using an amplified translational gain.

Another new line of research related to this topic could be the prompt detection and prevention of any accidents caused by VR sickness. The help of electroencephalogram (EEG) can help in the identification of cognitive conflict [Singh et al.2018], motion sickness [Chen et al. 2010], or even postural instability [Slobounov et al. 2009]. After the identification of an issue, the headset itself can produce a way to alert the user of the accident [Tirado Cortes etal. 2019] and help the user in recovering.

\section{CONCLUSION}

This paper presents the findings from an experiment investigating VR sickness and gait parameters during non-isometric virtual walking with large and perceivable translational gain. Most participants could accomplish the non-isometric virtual walking, even with large gains and without any prior training. However, overall, as the TG increased, participants reported higher VR sickness scores during the experiment. Changes in gait performance and 
CoM displacement were most prominent when the participants first experienced amplified virtual walking at $2 x$ TG. However, the gait performance seemed to stabilize and remain relatively stable after $2 X \mathrm{TG}$, and there were few significant differences detected among higher TGs $\{4 x, 6 x, 8 x, 10 x\}$ Surprisingly, participants with lower post-experiment SSQ scores adopted to the virtual walking with large TGs very well and even started showing gait performance improvement, even at large translational gain levels.

\section{ACKNOWLEDGMENTS}

This work was supported in part by the Australian Research Council (ARC) under discovery grant DP180100670 and DP180100656. We also thank the NSW Defence Innovation Network and NSW State Government of Australia for financial support of this project through grant DINPP2019 S1-03/09. Research was also sponsored in part by the Office of Naval Research Global, US, and was accomplished under Cooperative Agreement Number ONRG - NICOP N62909-19-1-2058.

\section{REFERENCES}

Philipp Agethen, Viswa Subramanian Sekar, Felix Gaisbauer, Thies Pfeiffer, Michael Otto, and Enrico Rukzio. 2018. Behavior Analysis of Human Locomotion in the Real World and Virtual Reality for the Manufacturing Industry. ACM Trans. Appl Percept. 15,3, Article 20 (July 2018), 19 pages. https://doi.org/10.1145/3230648

F. Argelaguet. 2014. Adaptive navigation for virtual environments. In 2014 IEEE Symposium on 3D User Interfaces (3DUI). 123-126. https://doi.org/10.1109/3DUI. 2014.7027325

Domna Banakou, Raphaela Groten, and Mel Slater. 2013. Illusory ownership of a virtual child body causes overestimation of object sizes and implicit attitude changes. Proceedings of the National Academy of Sciences 110, 31 (2013), 12846-12851. https://doi.org/10.1073/pnas.1306779110 arXiv:https://www.pnas.org/content/110/31/12846.full.pdf

Dominikus Baur, Sebastian Boring, and Steven Feiner. 2012. Virtual Projection: Exploring Optical Projection As a Metaphor for Multi-device Interaction. In Proceedings of the SIGCHI Conference on Human Factors in Computing Systems (CHI'12). ACM, New York, NY, USA, 1693-1702. https://doi.org/10.1145/2207676.2208297

Evren Bozgeyikli, Andrew Raij, Srinivas Katkoori, and Rajiv Dubey. 2016. Point \& Teleport Locomotion Technique for Virtual Reality. https://doi.org/10.1145/2967934. 2968105

Michael J. Cevette, Jan Stepanek, Daniela Cocco, Anna M. Galea, Gaurav N. Pradhan, Linsey S. Wagner, Sarah R. Oakley, Benn E. Smith, David A. Zapala, and Kenneth H. Brookler. 2012. Oculo-Vestibular Recoupling Using Galvanic Vestibular Stimulation to Mitigate Simulator Sickness. Aviation Space and Environmental Medicine (2012) https://doi.org/10.3357/ASEM.3239.2012

Jean Rémy Chardonnet, Mohammad Ali Mirzaei, and Frédéric Mérienne. 2017. Features of the PosturalSway Signal as Indicators to Estimate and PredictVisually Induced Motion Sickness in Virtual Reality. International Journal of Human-Computer Interaction (2017). https://doi.org/10.1080/10447318.2017.1286767

Yu Chieh Chen, Jeng Ren Duann, Shang Wen Chuang, Chun Ling Lin, Li Wei Ko, Tzyy Ping Jung, and Chin Teng Lin. 2010. Spatial and temporal EEG dynamics of motion sickness. NeuroImage 49, 3 (2010), 2862-2870. https://doi.org/10.1016/j. neuroimage.2009.10.005

J W Clauser, Charles E ; McConville, John T ; Young. 1969. WEIGHT, VOLUME, AND CENTER OF MASS OF SEGMENTS OF THE HUMAN BODY. Technical Report ANTIOCH COLL YELLOW SPRINGS OH. 112 pages. https://apps.dtic.mil/dtic/tr/ fulltext/u2/710622.pdf

Simon Davis, Keith Nesbitt, and Eugene Nalivaiko. 2014. A Systematic Review of Cybersickness. Proceedings of the 2014 Conference on Interactive Entertainment IE2014 (2014), 1-9. https://doi.org/10.1145/2677758.2677780

MarkS. Dennison, A. ZacharyWisti, and Michael D'Zmura. 2016. Use of physiologica signals to predict cybersickness. Displays 44 (2016), 42-52. https://doi.org/10.1016/ j.displa.2016.07.002

Natalia Duzmanska, Pawel Strojny, and Agnieszka Strojny. 2018. Can simulator sickness be avoided? A review on temporal aspects of simulator sickness. Frontiers in Psychology 9, NOV (2018). https://doi.org/10.3389/fpsyg.2018.02132

Ajoy S. Fernandes and Steven K. Feiner. 2016. Combating VR sickness through subtle dynamic field-of-view modification. 2016 IEEE Symposium on 3D User Interfaces, 3DUI 2016 - Proceedings (2016), 201-210. https://doi.org/10.1109/3DUI.2016.7460053 Germán Gálvez-García, Marion Hay, and Catherine Gabaude. 2015. Alleviating simulator sickness with galvanic cutaneous stimulation. Human Factors (2015). https://doi.org/10.1177/0018720814554948

JE Garrett, J Mulder, and A Veale. 1988. Trends in the use of an urban accident and emergency department by asthmatics. The New Zealand medical journal 101, 846 (May 1988), 253-255.http://europepmc.org/abstract/MED/3374895

Timofey Grechkin, Jerald Thomas, Mahdi Azmandian, Mark Bolas, and Evan Suma. 2016. Revisiting detection thresholds for redirected walking. In Proceedings of the ACM Symposium on Applied Perception - SAP'16. https://doi.org/10.1145/2931002. 2931018

Alan Hreljac and Robert N Marshall. 2000. Algorithms to determine event timing during normal walking using kinematic data. Journal of Biomechanics 33, 6 (jun 2000), 783-786. https://doi.org/10.1016/S0021-9290(00)00014-2

V. Interrante, L. Anderson, and B. Ries. 2007. Seven League Boots: A New Metaphor for Augmented Locomotion through Moderately Large Scale Immersive Virtual Environments. In 2007 IEEE Symposium on 3D UserInterfaces(3DUI), Vol. 00. null. https://doi.org/10.1109/3DUI.2007.340791

H. Iwata, H. Yano, H. Fukushima, and H. Noma. 2005. CirculaFloor [locomotion interface]. IEEE Computer Graphics and Applications 25, 1 (Jan 2005), 64-67. https: //doi.org/10.1109/MCG.2005.5

Omar Janeh, Gerd Bruder, Frank Steinicke, Alessandro Gulberti, and Monika PoetterNerger. 2017a. Analyses of Gait Parameters of Younger \& Older Adults during (Non-)Isometric Virtual Walking. IEEE Transactions on Visualization and Computer Graphics 24, 10 (2017), 2663-2674. https://doi.org/10.1109/TVCG.2017.2771520

O Janeh, E Langbehn, F Steinicke, G Bruder, A Gulberti, and M Poetter-Nerger. 2017b. Walking in virtual reality: Effects of manipulated visual self-motion on walking biomechanics. ACM Transactions on Applied Perception (2017). https://doi.org/10. $1145 / 3022731$

Robert S. Kennedy, Norman E. Lane, Kevin S. Berbaum, and Michael G. Lilienthal. 1993. Simulator Sickness Questionnaire: An Enhanced Method for Quantifying Simulator Sickness. The International Journal of Aviation Psychology 3,3 (1993), 203-220. https://doi.org/10.1207/s15327108ijap0303_3

D Lafond, M Duarte, and F Prince. 2004. Comparison of three methods to estimate the center of mass during balance assessment. Journal of Biomechanics 37,9 (sep 2004), 1421-1426. https://doi.org/10.1016/S0021-9290(03)00251-3

Eike Langbehn, Paul Lubos, Gerd Bruder, and Frank Steinicke. 2017. Bending the Curve: Sensitivity to Bending of Curved Paths and Application in Room-Scale VR. IEEE Transactions on Visualization and Computer Graphics 23, 4 (2017), 1349-1358. https://doi.org/10.1109/TVCG.2017.2657220

Joseph J. LaViola. 2000. A discussion of cybersickness in virtual environments. ACM SIGCHI Bulletin 32, 1 (2000), 47-56. https://doi.org/10.1145/333329.333344

Chin Teng Lin, Shu Fang Tsai, and Li Wei Ko. 2013. EEG-based learning system for online motion sickness level estimation in a dynamic vehicle environment. IEEE Transactions on Neural Networks and Learning Systems 24, 10 (2013), 1689-1700. https://doi.org/10.1109/TNNLS.2013.2275003

Morgan McCullough, Hong Xu, Joel Michelson, Matthew Jackoski, Wyatt Pease, William Cobb, William Kalescky, Joshua Ladd, and Betsy Williams. 2015. Myo Arm: Swinging to Explore a VE. In Proceedings of the ACM SIGGRAPH Symposium on Applied Perception (SAP '15). ACM, New York, NY, USA, 107-113. https://doi.org/10.1145/2804408.2804416

Niels Christian Nilsson, Stefania Serafin, and Rolf Nordahl. 2013. The Perceived Naturalness of Virtual Locomotion Methods Devoid of Explicit Leg Movements. In Proceedings of Motion on Games (MIG '13). ACM, New York, NY, USA, Article 133, 10 pages. https://doi.org/10.1145/2522628.2522655

Niels Christian Nilsson, Stefania Serafin, and Rolf Nordahl. 2014. Establishing the range of perceptually natural visual walking speeds for virtual walking-in-place locomotion. IEEE Transactions on Visualization and Computer Graphics 20, 4 (apr 2014), 569-578. https://doi.org/10.1109/TVCG.2014.21

Niels Christian Nilsson, Stefania Serafin, Frank Steinicke, and Rolf Nordahl. 2018. Natural Walking in Virtual Reality. Computers in Entertainment 16, 2 (2018), 1-22. https://doi.org/10.1145/3180658

Stephen Palmisano, Robert S. Allison, Juno Kim, and Frederick Bonato. 2011. Simulated Viewpoint Jitter Shakes Sensory Conflict Accounts of Vection. Seeing \& Perceiving 24, 2 (2011), 173 - 200. https://www-lib-uts-edu-au.ezproxy.lib.uts.edu.au/goto?

Anjul Patney, Joohwan Kim, Marco Salvi, Anton Kaplanyan, Chris Wyman, Nir Benty, Aaron Lefohn, and David Luebke. 2016. Perceptually-based Foveated Virtual Reality. In ACM SIGGRAPH 2016 Emerging Technologies (SIGGRAPH '16). ACM, New York, NY,USA, Article 17, 2 pages. https://doi.org/10.1145/2929464.2929472

E. D. Ragan, S. Scerbo, F. Bacim, and D. A. Bowman. 2017. Amplified Head Rotation in Virtual Reality and the Effects on 3D Search, Training Transfer, and Spatial Orientation. IEEE Transactions on Visualization and Computer Graphics 23, 8 (Aug 2017), 1880-1895. https://doi.org/10.1109/TVCG.2016.2601607

Lisa Rebenitsch and Charles Owen. 2016. Review on cybersickness in applications and visual displays. Virtual Reality 20,2 (2016),101-125. https://doi.org/10.1007/s10055016-0285-9

Gary E. Riccio and Thomas A. Stoffregen. 1991. An Ecological Theory of Motion Sickness and Postural Instability. Ecological Psychology (1991). https://doi.org/10. 1207/s15326969eco0303_2

A. Fleming Seay, David M. Krum, Larry Hodges, and William Ribarsky. 2002. Simulator Sickness and Presence in a High Field-of-view Virtual Environment. In CHI 'O2 
Extended Abstracts on Human Factors in Computing Systems (CHI EA '02). ACM, New York, NY, USA, 784-785. https://doi.org/10.1145/506443.506596

Avinash Kumar Singh, Hsiang Ting Chen, Yu Feng Cheng, Jung Tai King, Li Wei Ko, Klaus Gramann, and Chin Teng Lin. 2018. Visual Appearance Modulates Prediction Error in Virtual Reality. IEEE Access (2018). https://doi.org/10.1109/ACCESS.2018. 2832089

Mel Slater, Daniel Perez-Marcos, H. Henrik Ehrsson, and Maria V. Sanchez-Vives. 2009. Inducing illusory ownership of a virtual body. Frontiers in Neuroscience 3, SEP (2009), 214-220. https://doi.org/10.3389/neuro.01.029.2009

Mel Slater, Martin Usoh, and Anthony Steed. 1995. Taking Steps: The Influence of a Walking Technique on Presence in Virtual Reality. ACM Trans. Comput.-Hum Interact. 2, 3 (Sept. 1995), 201-219. https://doi.org/10.1145/210079.210084

Semyon Slobounov, Cheng Cao, Niharika Jaiswal, and Karl M. Newell. 2009. Neural basis of postural instability identified by VTC and EEG. Experimental Brain Research 199,1 (01 Oct 2009), 1-16. https://doi.org/10.1007/s00221-009-1956-5

Kay Stanney and Robert Kennedy. 2010. Simulation Sickness. In Human Factors in Simulation and Training. https://doi.org/10.1201/9781420072846.ch6

Kay M Stanney, Robert S Kennedy, and Julie M Drexler. 1997. Cybersickness is not simulator sickness. Proceedings of the Human Factors and Ergonomics Society 41st Annual Meeting (1997), 1138-1142.https://doi.org/10.1177/107118139704100292

F Steinicke, G Bruder, J Jerald, H Frenz, and M Lappe. 2010. Estimation of Detection Thresholds for Redirected Walking Thechniques. Ieee Tvcg 16, 1 (jan 2010), 17-27. https://doi.org/10.1109/TVCG.2009.62

E.A.Suma,Z. Lipps, S. Finkelstein, D. M. Krum, and M.Bolas. 2012. Impossible Spaces: Maximizing Natural Walking in Virtual Environments with Self-Overlapping $\mathrm{Ar}$ chitecture. IEEE Transactionson Visualization and Computer Graphics 18, 4 (April 2012), 555-564. https://doi.org/10.1109/TVCG.2012.47

Qi Sun, Li-Yi Wei, and Arie Kaufman. 2016. Mapping Virtual and Physical Reality. ACM Trans. Graph. 35, 4, Article 64 (July 2016), 12 pages. https://doi.org/10.1145/ 2897824.2925883

D. Swapp, J. Williams, and A. Steed. 2010. The implementation of a novel walking interface within an immersive display. In 2010 IEEE Symposium on 3D User Interfaces (3DUI). 71-74. https://doi.org/10.1109/3DUI.2010.5444717

C. A. Tirado Cortes, H. Chen, D. Sturnieks, J. Garcia Marin, S. Lord, and C. Lin. 2019 Evaluating Balance Recovery Techniques for Users Wearing Head-Mounted Display in VR. IEEE Transactions on Visualization and Computer Graphics (2019), 1-1.
https://doi.org/10.1109/TVCG.2019.2927477

Sam Tregillus, Majed Al Zayer, and Eelke Folmer. 2017. Handsfree Omnidirectional VR Navigation Using Head Tilt. In Proceedings of the 2017 CHI Conference on Human Factors in Computing Systems (CHI '17). ACM, New York, NY, USA, 4063-4068. https://doi.org/10.1145/3025453.3025521

Sam Tregillus and Eelke Folmer. 2016. VR-STEP: Walking-in-Place Using Inertial Sensing for Hands Free Navigation in Mobile VR Environments. In Proceedings of the 2016 CHI Conference on Human Factors in Computing Systems (CHI '16). ACM, New York, NY, USA, 1250-1255. https://doi.org/10.1145/2858036.2858084

Martin Usoh, Kevin Arthur, Mary C. Whitton, Rui Bastos, Anthony Steed, Mel Slater, and Frederick P. Brooks. 1999. Walking $>$ walking-in-place $>$ flying, in virtual environments. In Proceedings of the 26th annual conference on Computer graphics and interactive techniques -SIGGRAPH'99. ACM Press, New York, New York, USA, 359-364. https://doi.org/10.1145/311535.311589 arXiv:15334406

T.Weißker, A. Kunert, B. Fröhlich, and A. Kulik. 2018. Spatial Updating and Simulator Sickness During Steering and Jumping in Immersive Virtual Environments. In 2018 IEEE Conference on Virtual Reality and 3D User Interfaces (VR). 97-104. https: //doi.org/10.1109/VR.2018.8446620

Graham Wilson, Mark McGill, Matthew Jamieson, Julie R. Williamson, and Stephen A. Brewster. 2018. Object Manipulation in Virtual Reality Under Increasing Levels of Translational Gain. Proceedings of the 2018 CHI Conference on Human Factors in Computing Systems - CHI'18(2018),1-13. https://doi.org/10.1145/3173574.3173673

Marjorie Woollacott and Anne Shumway-Cook. 2002. Attention and the control of posture and gait: a review of an emerging area of research. Gait and Posture 16, 1 (2002), 1 - 14. https://doi.org/10.1016/S0966-6362(01)00156-4

Galit Yogev-Seligmann, Jeffrey M. Hausdorff, and Nir Giladi. 2008. The role of executive function and attention in gait. https://doi.org/10.1002/mds.21720 arXiv:NIHMS150003

John K.Zao, Tzyy Ping Jung, Hung Ming Chang, Tchin Tze Gan, Yu TeWang, Yuan Pin Lin, Wen Hao Liu, Guang Yu Zheng, Chin Kuo Lin, Chia Hung Lin, Yu Yi Chien, Fang Cheng Lin, Yi Pai Huang, Sergio José Rodríguez Méndez, and Felipe A. Medeiros. 2016. Augmenting VR/AR applications with EEG/EOG monitoring and oculo-vestibular recoupling. In Lecture Notes in Computer Science (including subseries Lecture Notes in Artificial Intelligence and Lecture Notes in Bioinformatics). https://doi.org/10.1007/978-3-319-39955-3_12 